\title{
A process model for air bending
}

\author{
L.J. de Vin ${ }^{a, b, *}$, A.H. Streppel ${ }^{b}$, U.P. Singh ${ }^{a}$, H.J.J. Kals ${ }^{b}$ \\ ${ }^{a}$ University of Ulster at Jordanstown, Jordanstown, Northern Ireland, UK \\ ${ }^{\mathrm{b}}$ Department of Mechanical Engineering, University of Twente, PO Box 217, 7500 AE Enschede, Netherlands
}

Received 1 August 1994

\section{Industrial summary}

A so called 'three-section' model for air bending is presented. It is assumed that a state of plane strain exists and that Bernoulli's law is valid. The material behaviour is described with Swift's equation, and the change of Young's modulus under deformation is addressed. As compared with other models, the model described in the paper is capable of generating information such as required punch displacement and the unfolded blank size, very accurately. With in-process measurement of the spring-back angle, the punch displacement can be calculated even more accurately.

\section{Introduction}

In the small-batch-part manufacturing of sheet metal components, manufacturing departments are facing decreasing batch sizes, an increasing variety of parts and increasing accuracy demands. This holds especially for sub-contractors, since these companies have to produce parts for different customers which are used for totally different applications. As a consequence, standardization of bend radii, sheet material and thicknesses can not be obtained. As a result of the variety of parts, tool change times can become a problem, as indicated by Kroeze [1].

The air bending process offers the advantage that many less tool changes are required as compared with bottoming. However, the calculation of the unfolded blank size and of the required punch displacement presents some problems. The lack of adequate process models hampers the industrial use of air bending in many cases, and in other cases companies have even abandoned the use of air bending for this reason.

The increasing accuracy-demands present an additional problem. As a result of these demands, in bending, the bend angle obtained must lie within a very narrow range. Due to variations in the sheet thickness and the mechanical properties of the sheet material, this can be a problem. Especially, the influence of the aforementioned variations on the spring-back angle presents a problem. In bottoming, the required bend angle is often obtained with a time consuming trial-and-error method. During this trial-and-error cycle, tool changes may be

* Corresponding author. required, since the bend angle under loading conditions is determined completely by the geometry of the tools, and the spring-back angle is predominantly determined by the mechanical behaviour and the thickness of the sheet: the process force has only a minor influence on the spring-back angle. As a result, for a given material and sheet thickness, only a very limited range of bend angles can be obtained with the use of one tool set. The air bending process offers the advantage that a large range of bend angles can be obtained with one set of tools. Provided that the sheet geometry under loading can be predicted adequately, in-process control of the springback angle can result in better accuracy.

\section{Process models for air bending}

A simple model for air bending is the rigid-plastic model. In this model, the sheet geometry is described by a circular section and two straight sections, and there is no spring-back. This type of model is often used as a reference to describe the effect of spring-back and other phenomena on the sheet geometry. The sheet geometry for a rigid-plastic model is shown in Fig. 1.

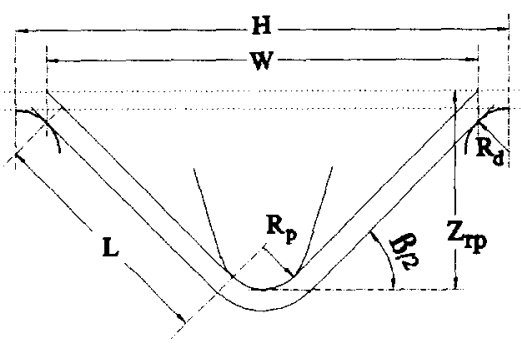

Fig. 1. Rigid-plastic model. 


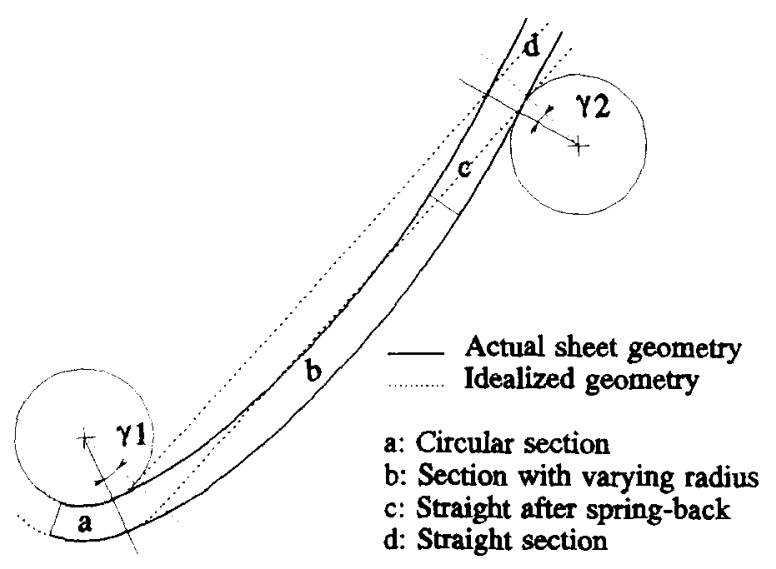

Fig. 2. Sheet geometry according to a 'three section' model.

When the rigid-plastic model is extended with the spring-back effect, a so called 'circular-straight' model is obtained. According to such a model, the sheet geometry after unloading consists of two straight sections and a circular section. Under loading conditions, these sections are also deformed elastically, which results in a larger required punch displacement when compared to a rigid-plastic model.

Basically different models are referred to here as 'three section' models. In these models, three types of deformed sections can be determined in a bent sheet under loading conditions: (i) a wrap-around zone under the punch, which has an inner radius equal to the punch radius; (ii) two elasto-plastically deformed zones in which the local bend radius varies; and (iii) two elastically-deformed only sections. For monolithic sheet, the sheet material on the outside of the die shoulders remains straight. The corresponding sheet geometry is shown in Fig. 2. Other models in which the influence of the section with varying radius is addressed are described by Stelson [2] and by Wang [3].

\section{Material behaviour}

The description of the material behaviour is very important in a three-section model (Streppel [4]). In this paper, it is assumed that a state of plane strain exists and that Bernoulli's law is valid (see also Fig. 3):

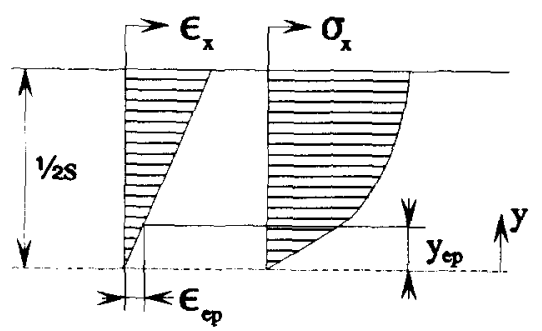

Fig. 3. Strain according to Bernoulli's law, stress according to Hooke's Law and Swift's equation.
$\varepsilon_{x}=\frac{y}{R_{m}}$

Elastic behaviour according to Hooke's law can be written as follows for a state of plane strain:

$\sigma_{x}=E^{\prime} \varepsilon_{x} \quad$ with $\quad E^{\prime}=\frac{E}{1-v^{2}}$

As the stress-strain relationship for plastic deformation, Swift's equation (sometimes referred to as extended Ludwik - Nadai) is adopted here:

$\sigma_{x}=C^{\prime}\left(\delta_{x}+\varepsilon_{0}\right)^{n}$ with $C^{\prime}=\left(\frac{4}{3}\right)^{(n+1) 2} C$

With these adoptions, the relationship between the local bending moment and the local curvature can be determined, as described in Section 3.1.

An important complication is that Young's modulus decreases under deformation, as found, for instance, by Lems [5]. This results in a larger spring-back angle. This effect is addressed in Section 3.2.

\subsection{The relation between the local bending moment and the local bend radius}

The bending moment can be split into an elastic contribution and an elastoplastic contribution and is calculated from:

$$
\begin{aligned}
& M=M_{\mathrm{el}}+M_{\mathrm{pl}}, \quad M_{\mathrm{el}}=2 \cdot \int_{0}^{\gamma_{\mathrm{ep}}} E^{\prime} \varepsilon_{x} y \mathrm{~d} y . \\
& M_{\mathrm{pl}}=2 \cdot \int_{\mathrm{ep}^{\prime}}^{s / 2} C^{\prime}\left(\delta_{x}+\varepsilon_{0}\right)^{n} y \mathrm{~d} y
\end{aligned}
$$

According to Bernoulli, $\varepsilon_{X}$ equals $y / R_{\mathrm{m}}$ and Eq. (4) can be written as:

$$
\begin{aligned}
& M_{\mathrm{el}}=\frac{2}{3} \frac{E^{\prime}}{R_{m}} y_{\mathrm{ep}}^{3}=\frac{2}{3} E^{\prime} R_{m}^{2} \varepsilon_{\mathrm{ep}}^{3}, \\
& M_{\mathrm{pl}}=2 \cdot \int_{y_{\mathrm{ep}}}^{\mathrm{s}_{2} 2} C^{\prime}\left(\frac{y}{R_{m}}+\varepsilon_{0}\right)^{\prime \prime} y \mathrm{~d} y
\end{aligned}
$$

The elasto-plastic contribution can be calculated after the following substitution:

$\left(\frac{y}{R_{m}}+\varepsilon_{w}\right)=V$ and thus $\mathrm{d} y=R_{m} \mathrm{~d} V$

For the elasto-plastic contribution to the bending moment, Eqs 5 and 6 yield:

$$
\begin{aligned}
M_{\mathrm{pl}} & =2 \cdot \int_{V_{\min }}^{V_{\max }} C^{\prime} V^{n}\left(V-\varepsilon_{0}\right) R_{m}^{2} \mathrm{~d} V \\
& =2 \cdot C^{\prime} R_{m \prime}^{2} \int_{V_{\text {min }}}^{V_{\text {max }}^{\prime}}\left(V^{n+1}-\varepsilon_{01} V^{n}\right) \mathrm{d} V
\end{aligned}
$$




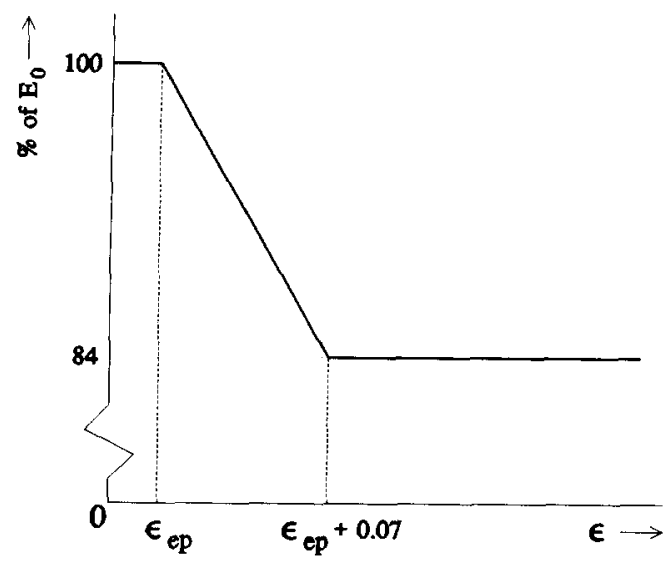

Fig. 4. A simple model for the change of Young's modulus.

where

$V_{\min }=\frac{y_{\mathrm{ep}}}{R_{m}}+\varepsilon_{0} \quad$ and $\quad V_{\max }=\frac{s}{2 R_{m}}+\varepsilon_{0}$

With $V_{\min }$ and $V_{\max }$ as defined above, Eq. (7) yields:

$$
\begin{aligned}
M_{\mathrm{pl}}= & 2 \cdot C^{\prime} R_{m}^{2}\left[\frac{1}{n+2}\left(V_{\max }^{n+2}-V_{\min }^{n+2}\right)\right. \\
& \left.-\frac{\varepsilon_{0}}{n+1}\left(V_{\max }^{n+1}-V_{\min }^{n+1}\right)\right]
\end{aligned}
$$

The last equation makes it possible to determine the local curvatures of the sheet under loading conditions. This forms the basis of the three section model discussed in this paper, and reported previously by De Vin $[6,7]$.

\subsection{The influence of the changing Young's modulus on the spring-back}

Due to the change of Young's modulus of elasticity $\left(E^{\prime}\right)$ under deformation, the spring-back is larger than the spring-back calculated with a constant value of $E^{\prime}$. A simple model for the change of $E^{\prime}$ is shown in Fig. 4, this model being based on data collected by Brinkman [8].

When plastic deformation occurs $\left(\varepsilon_{x}>\varepsilon_{\mathrm{ep}}\right)$, the change in $E^{\prime}$ can be accounted for by redefining $E^{\prime} I$ according to Eq. (10):

$E^{\prime} I=\int_{-s / 2}^{s / 2} E^{\prime}\left(\varepsilon_{x}\right) y^{2} d y$

To calculate $E^{\prime} I$, this integration must be split into three parts:

$\left[E^{\prime}\right]_{1}=2 \cdot \int_{0}^{y_{\mathrm{ep}}} E_{0}^{\prime} y^{2} \mathrm{~d} y=\frac{2}{3} y_{\mathrm{ep}}^{3} E_{0}^{\prime}$
$\left[E^{\prime} I\right]_{2}=2 \cdot \int_{y_{\mathrm{ep}}}^{y^{* *}} E_{0}^{\prime}\left[1-\left(\frac{y}{R}-\varepsilon_{\mathrm{ep}}\right) \cdot \frac{0.16}{0.07}\right] y^{2} \mathrm{~d} y$

$\left[E^{\prime} T\right]_{3}=2 \cdot \int_{y^{*}}^{s / 2} 0.84 \cdot E_{0}^{\prime} y^{2} \mathrm{~d} y$

where $y^{*}$ is defined by Eq. (14):

$y^{*}=\min \left(s / 2,\left(0.07+\varepsilon_{\mathrm{ep}}\right) \cdot R_{\mathrm{m}}\right)$

and:

$E^{\prime} I=\left[E^{\prime} I\right]_{1}+\left[E^{\prime} I\right]_{2}+\left[E^{\prime} \Pi_{3}\right.$

Note that $\left[E^{\prime} I\right]_{3}=0$ when $y^{*}=s / 2$. With:

$y_{\mathrm{ep}}=\varepsilon_{\mathrm{ep}} \cdot R_{m}$

and $y^{*}$ as defined in Eq. (14), the results of the integrations can be expressed in $R_{m}, E_{0}^{\prime}, s$ and $\varepsilon_{\mathrm{ep}}$. For a given sheet, $E_{0}^{\prime}, s$ and $\varepsilon_{\mathrm{ep}}$ have a fixed value and $R_{m}$ is the only variable. Solving the integrals 11,12 and 13 yields:

$\left[E^{\prime}\right]_{1}=\frac{2}{3}\left(\varepsilon_{\mathrm{ep}} \cdot R_{m}\right)^{3} E_{0}^{\prime}$

$$
\begin{aligned}
{\left[E^{\prime} I\right]_{2}=} & {\left[\left(\frac{2}{3}+\frac{0.32}{0.21} \varepsilon_{\mathrm{ep}}\right) \cdot\left(\left(y^{*}\right)^{3}-\left(\varepsilon_{\mathrm{ep}} \cdot R_{m}\right)^{3}\right)\right.} \\
& \left.-\frac{0.32}{0.28 R_{m}} \cdot\left(\left(y^{*}\right)^{4}-\left(\varepsilon_{\mathrm{ep}} \cdot R_{m}\right)^{4}\right)\right] \cdot E_{0}^{\prime} \\
{\left[E^{\prime} I\right]_{3}=} & \frac{1.68}{3}\left(\frac{s^{3}}{8}-\left(y^{*}\right)^{3}\right) \cdot E_{0}^{\prime}
\end{aligned}
$$

With these equations, the spring-back and the local sheet curvature after unloading can be calculated.

\section{Experimental results versus calculated results}

Experiments have been carried out with cold rolled steel, with a sheet thickness of 1,2 and $3 \mathrm{~mm}$ and a sheet width of $40 \mathrm{~mm}$. The punch used is a Modufix BX-4 punch with a radius of $3 \mathrm{~mm}$. A conventional die and three Modufix ${ }^{B}$ dies have been used, as listed in Table 1.

With the length correction as defined in Fig. 5, the results are listed in Fig. 6. The calculated values are much more accurate than the commonly used standard values. The calculated (absolute) length corrections are too low, except for a thickness of $3 \mathrm{~mm}$. A possible

Table 1

The geometry of the dies

\begin{tabular}{lll}
\hline Die id & H (mm) & Rd $(\mathrm{mm})$ \\
\hline OZ-009 & 19.3 & 2.0 \\
OX-4 & 18.30 & 1.5 \\
OX-5 & 23.06 & 2.0 \\
OX-6 & 27.84 & 2.5 \\
\hline
\end{tabular}




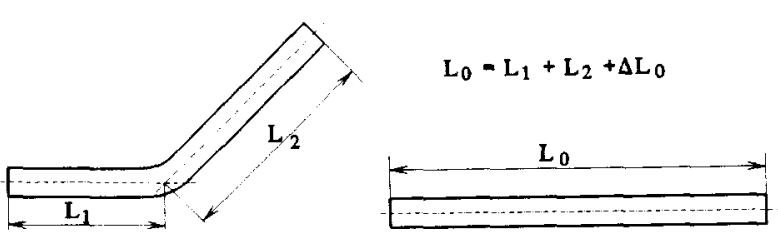

Fig. 5. Measured and calculated length correction (absolute values).

explanation is that the plane strain assumption may not be valid for this sheet thickness and bend radius in combination with the sheet width.

The calculated punch displacements are too low when compared with the experimental results. However, the calculated sensitivities of the bend angle to the punch displacement show extremely good correlation with the measured values (within $0.4 \%$ ). The calculated spring-back angles are significantly smaller than the measured spring-back angles for the complete set of experiments. This is remarkable, since the calculated spring-back angles are larger than those obtained from circular straight models, and the calculated bending moments show a very good correlation with the experimental values. A possible explanation is that Young's modulus may decrease by more than $16 \%$ or that the minimum value of the Young's modulus is reached at a lower value of strain.

When the measured spring-back angles instead of the calculated values are used in the three section model, the calculated punch displacements (indicated by ' $\mathrm{Cal}$ culated with correction' in Fig. 7) show a much better correlation with the experimental results. Therefore, in-process measurement of the spring-back angle can lead to significant improvements of accuracy in manufacturing. One possible way to measure the spring-back is to retract the punch partially at the end of the loading cycle and measuring the corresponding reduction of the punch force.

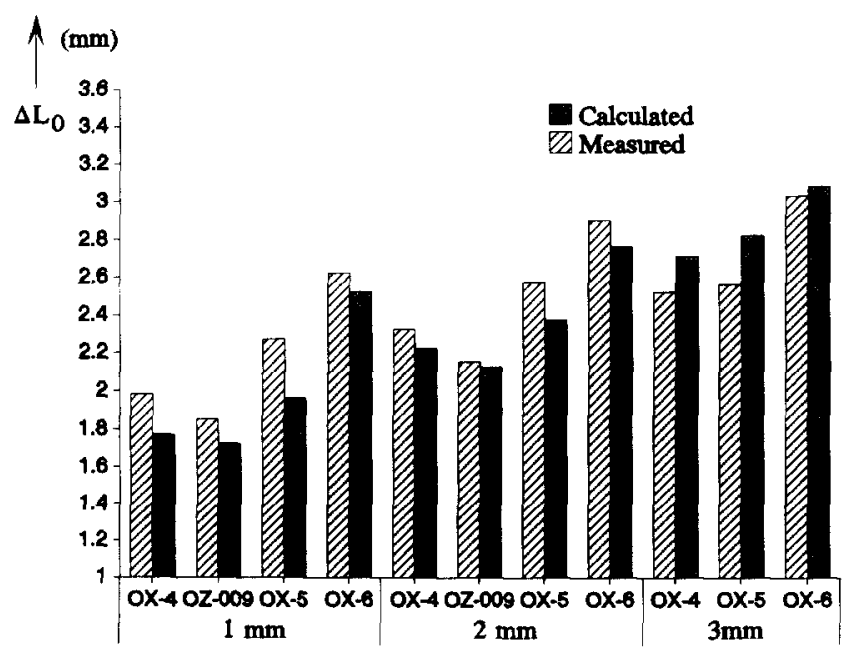

Fig. 6. The definition of the length correction $\Delta L_{0}$ for the bend allowance.

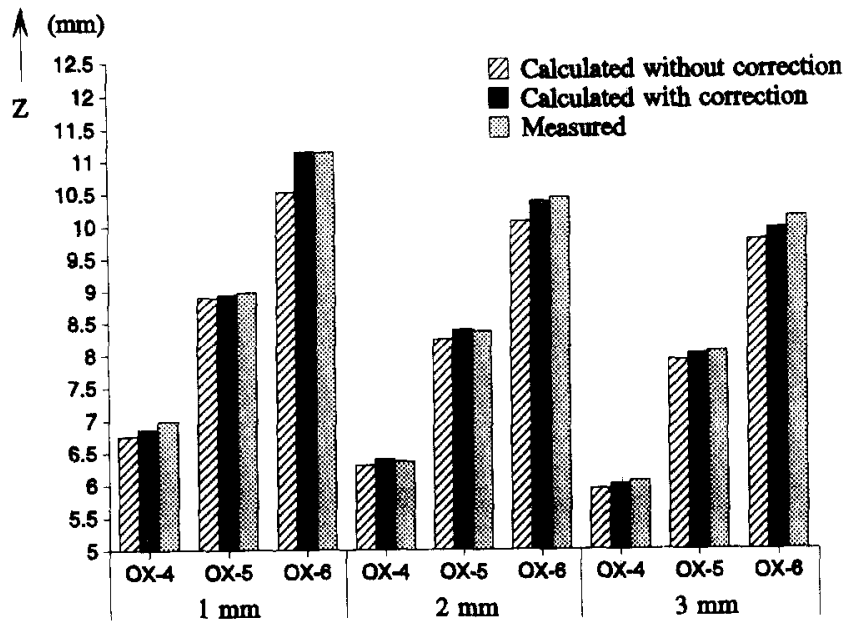

Fig. 7. Punch displacements.

\section{Simulations, carried out with the 'three section' model}

The model (described in more detail by De Vin in $[6,7])$ has been used to simulate the variation of the plane strain constant $C^{\prime}$ and the plane strain Young's modulus $E_{0}^{\prime}$, as well as the die-width $\mathrm{H}$. The influence on the required punch displacement and the unfolded blank size is discussed in Section 5.1. The variation of the strain hardening coefficient $n$ is discussed in Section 5.2 .

\subsection{The variation of $C^{\prime}, E_{0}^{\prime}$ and $H$}

It has been found that $C^{\prime}$ and $E_{0}^{\prime}$ have virtually no influence on the unfolded blank size, whereas the diewidth $\mathrm{H}$ has a significant influence. As a result, variations of the mechanical properties of the sheet material are negligible. An important consequence of the influence of $H$ is that the tool geometry must be known before the unfolded blank size of a part can be calculated. The simulation results are shown in Fig. 8. The corresponding length correction calculated with the ASM and DIN standards is approx. $-1.4 \mathrm{~mm}$ (for cold rolled steel with a bend angle of $90^{\circ}$, a sheet thickness of $1 \mathrm{~mm}$ and an inner bend radius of $3 \mathrm{~mm}$ ).

$C^{\prime}$ and $E_{0}^{\prime}$ have been found to influence the required punch displacement as shown in Fig. 9. Further research has established that this influence is caused mainly by the influence on the spring-back angle. For the representation of the influence of the die-width $H$, the required punch displacement was divided by the corresponding displacement, calculated with a rigidplastic model, as shown along the right-hand $Y$-axis. It must be noted that $Z / Z_{\mathrm{rp}}<1$. As a consequence, rigidplastic models tend to over-bend the parts, and circular-straight models do so even more. 


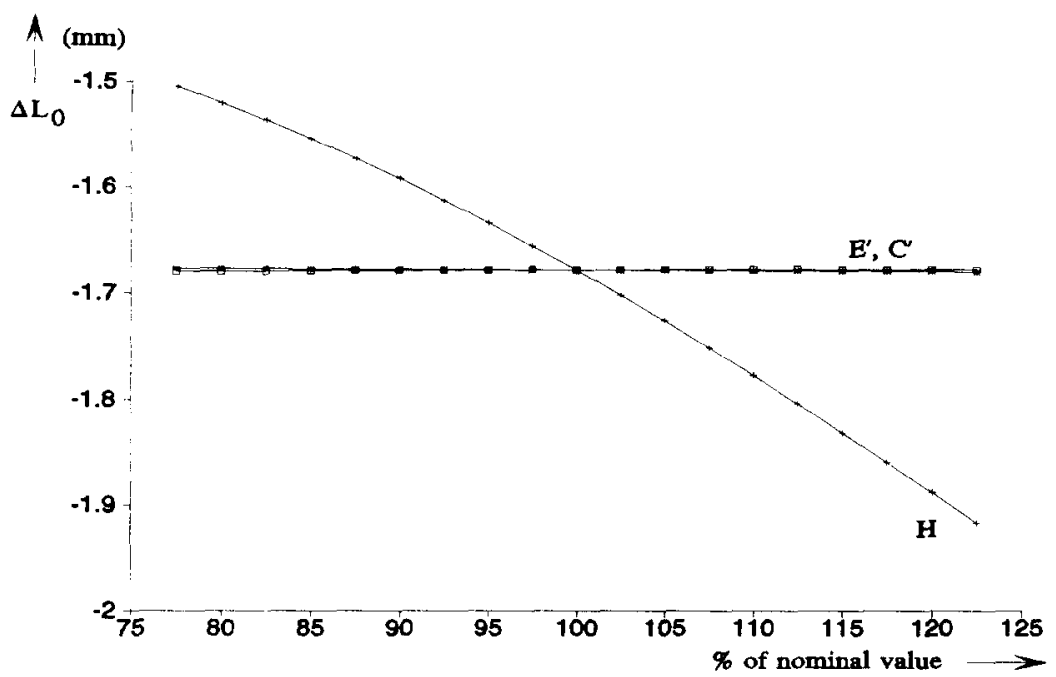

Fig. 8. The influence of $C^{\prime}, E_{0}^{\prime}$ and $H$ on the length correction.

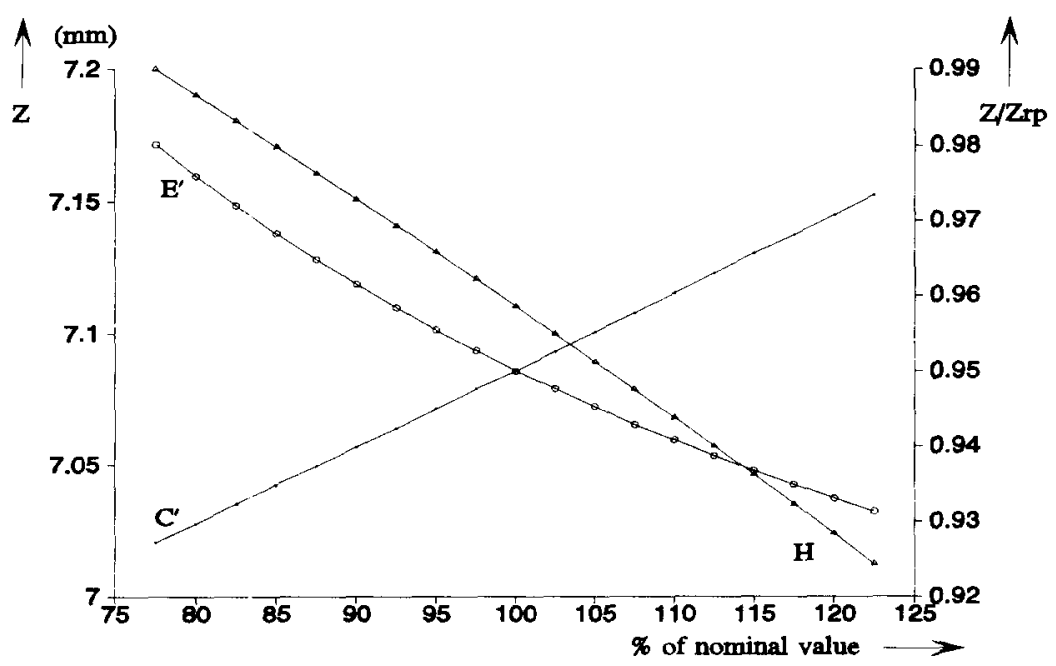

Fig. 9. The influence of $C^{\prime}, E_{0}^{\prime}$ and $H$ on the punch displacement.

\subsection{The variation of the strain hardening coefficient $n$}

In simulations of the bending process, often the material parameter $n$ is varied also. The purpose of variations of the strain-hardening coefficient $n$ is usually to investigate the influence of the strain-hardening. However, the actual strain hardening equals $\partial \sigma_{x} / \partial \varepsilon_{x}$ : it depends on the strain value whether an increase of $n$ results in greater or lesser strain hardening. Also, with a change in $n$, a number of effects is achieved simultaneously: (i) the strain hardening changes; (ii) the transition point between elastic and elasto-plastic behaviour moves; and (iii) the stress level changes. When, for instance, the value of $n$ is decreased, the stress level increases $\left(\varepsilon_{x}<1\right)$, the strain hardening for low strain values increases, the strain hardening for high strain values decreases and the elastic zone becomes larger.

Simulations have been carried out with the use of the bending model. Instead of just changing $n$, the parameters $C^{\prime}$ and $\varepsilon_{0}$ were changed also in such a way that the bending moment and the strain $\varepsilon_{\mathrm{ep}}$ at the transition between elastic and elasto-plastic behaviour remained the same. The simulations have been carried out for a sheet thickness of $2 \mathrm{~mm}$, an inner bend radius of $3 \mathrm{~mm}$ and the OX-5 die. The following results have been obtained (Table 2).

The spring-back angle $\Delta \alpha$ is virtually uninfluenced by variation of $n$ when the bending moment $M_{\mathrm{rp}}$ remains the same. Possibly, the change in $\Delta \alpha$ for different values of $n$ results completely from the differences in the punch displacement $(Z)$ for different values of $n$, the bend angle being $90^{\circ}$ for all three simulations. However, the position of the centre point of the circular section (indicated by $\delta Y$, see also Fig. 10) changes significantly. This indicates that the sheet geometry is different for the three values of $n$ and, as a result, the length 
Table 2

Results obtained for three values of $n$

\begin{tabular}{lcccl}
$n$ & 0.153 & 0.204 & 0.255 & {$[-]$} \\
$" o$ & 0.0016 & 0.0065 & 0.013 & {$[-]$} \\
$C^{\prime}$ & 564 & 615 & 667 & {$\left[\mathrm{~N} / \mathrm{mm}^{2}\right]$} \\
$M_{r p}$ & 424.6 & 424.5 & 424.8 & {$[\mathrm{Nmm} / \mathrm{mm}]$} \\
$\Delta x$ & 2.558 & 2.456 & 2.433 & {$[$ degrees] } \\
$i Y$ & 0.1495 & 0.1915 & 0.2234 & {$[\mathrm{~mm}]$} \\
$\Delta L_{0}$ & -2.310 & -2.367 & -2.408 & {$[\mathrm{~mm}]$} \\
$\mathrm{Z}$ & 8.403 & 8.222 & 8.068 & {$[\mathrm{~mm}]$} \\
\hline
\end{tabular}

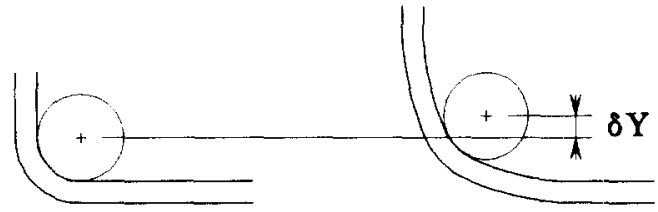

Fig. 10. The changed centre point position $\mathrm{d} Y$ of the circular section.

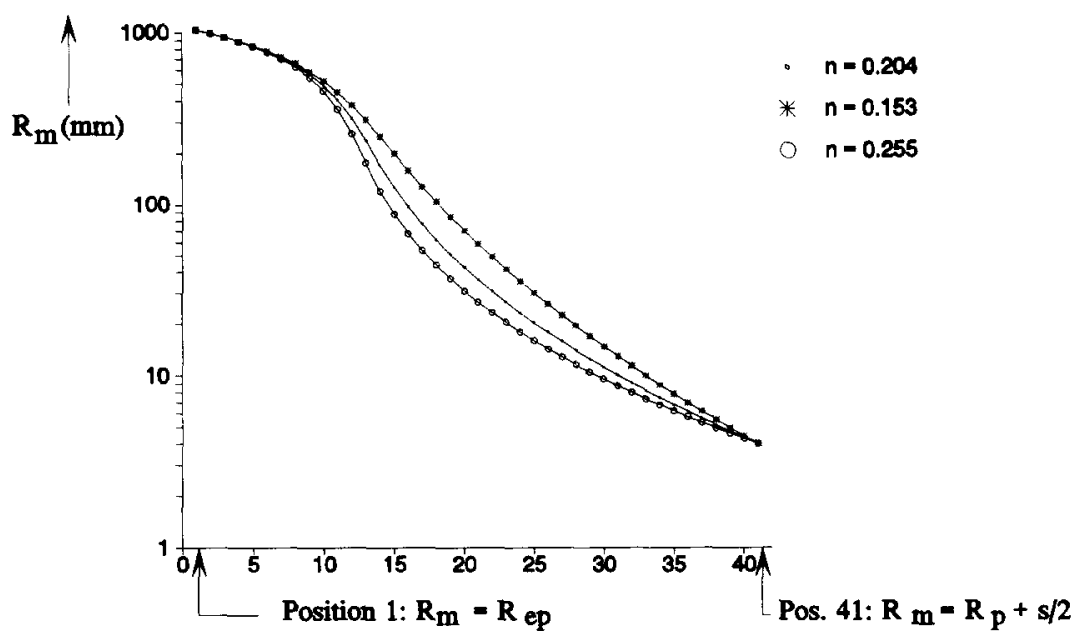

Fig. 11. The local bend radii.

correction $\Delta L_{0}$ is influenced as well. Furthermore, the required punch displacement $(Z)$ is influenced strongly also. This emphasizes the need to determine material parameters correctly. The changed sheet geometry is illustrated in Fig. 11, showing the local bend radii for three different values of $n$. At position no. 1, the bend radius at the transition point between elastic and elastoplastic deformation is shown, whilst position no. 41 represents the bend radius at the contact point between the sheet and the punch.

\section{Conclusions and recommendations}

The principles of a three section model for air bending have been discussed, and the equations describing the extended material model have been derived. It has been shown that the die-width influences the unfolded blank size of a part, which has major consequences, for instance in process planning. The calculated punch displacements show good correspondence with experimental results. Nevertheless, some improvements still seem to be possible. For instance, the in-process measurement of the spring-back angle can result in an improved accuracy of bent parts.
Future work by the authors will include the use of other models for the change of Young's modulus. The bending model will also be extended with a simple model for elastic-plastic recovery during spring-back.

\section{Nomenclature}

$C^{\prime}$ constant in the Ludwik-Nadai and Swift equation (plane strain equivalent of $C$ )

$E_{0}^{\prime} \quad$ Young's modulus for plane strain (nominal value)

$E_{0} \quad$ Nominal value of Young's modulus

$H$ die-width (centre to centre of the die shoulders)

$I$ moment of inertia

$M$ bending moment

$n \quad$ strain hardening coefficient

$R_{d} \quad$ radius of the die shoulders

$R_{m} \quad$ radius of the middle layer of a sheet

$R_{p} \quad$ punch radius

$s \quad$ sheet thickness

$W$ distance between the (instantaneous) contact points between the sheet and the die shoulders

$x \quad$ coordinate parallel with the sheet surface and perpendicular to the bend axis

$y \quad$ coordinate perpendicular to the sheet thickness

$Z$ punch displacement 


\section{Greek letters}

$\beta \quad$ bend angle

$\Delta L_{0} \quad$ length correction

$\delta Y$ change in the centre-point position of the circular section (for the 'three-section' model, compared with the rigid-plastic model)

$\varepsilon \quad$ strain

$\varepsilon_{0} \quad$ pre-strain

$v \quad$ Poisson's ratio of contraction

$\sigma \quad$ stress

\section{Subscripts}

el elastic

ep at the transition between elastic and elasto-plastic deformation

pl plastic

rp according to the rigid-plastic model

\section{References}

[1] B. Kroeze, A.H. Streppel and D. Lutters, Tools and accessories for press brakes, Proc. Shemet 94 Conf., Bellast. Northern Iretand 1994, pp 251-260.

[2] K.A. Stelson, An adaptive pressbrake control for strain hardening materials, J. Engng Ind., Trans. ASME, I08 (1986) 127132.

[3] C. Wang, G. Kinzel and T. Altan, Mathematical modeling of plane-strain bending of sheet and plate, J. Mater. Process. Technol., 39 (1993) 279-304.

[4] A.H. Streppel, L.J. De Vin, J. Brinkman and H.J.J. Kals, Suitability of sheet bending modelling techniques in CAPP applications. J. Mater. Process. Technol., 36 (1993) 339 356.

[5] W. Lems, The change of Young's modulus after deformation at low Temperature and its recovery, PhD Thesis, University of Delft. 1963.

[6] L.J. De Vin. A.H. Streppel, D. Lutters and H.J.J. Kals. A process model for air bending in CAPP applications, Proc. Shemet 94 Conf., Belfast, Northern Ireland, 1994. pp 17-. 28.

[7] L.J. De Vin, Computer aided process planning for the bending of sheet metal components, PhD Thesis, University of Twente, 1994.

[8] J. Brinkman, Sheet bending: The many aspects of a 'simple' forming process, Internal Report PR-369, University of Twente (in Dutch), 1990. 